\title{
Transverse Stability in Multibunch Mode for CLIC
}

\author{
G. Guignard \\ CERN, 1211 Geneva 23, Switzerland
}

\section{Abstract}

In order to reach the desire luminosity with $250 \mathrm{GeV}$ per beam, multibunch operation (limited to 4 bunches, say) might have to be considered in the CERN linear collider (CLIC). One limitation comes from the coupling of the bunch motion with the long-range transverse wake fields that may induce beam breakup. These wake fields have therefore to be controlled, and means of reducing their effects on the beam are discussed in a companion paper. One possibility consists in detuning the dipole modes in the cells to obtain decoherent contributions and hence reduce the field amplitude at the downstream bunch location. The important question is to know below which value this amplitude must be limited to prevent intolerable beam breakup. In a first attempt at estimating this threshold for CLIC two approaches are considered, i.e. the criterion developed at SLAC and based on the convergence of the multibunch-motion solution [1], and numerical simulations of two-bunch motion in a focusing lattice.

\section{INTRODUCTION}

Starting with the proposed parameters for the $500-\mathrm{GeV}$ CLIC option [2], i.e. $250 \mathrm{GeV}$ per beam, a single bunch with $6 \times 10^{9}$ particles in each linac leads to a total luminosity of $7.9 \times 10^{32} \mathrm{~cm}^{-2} \mathrm{~s}^{-1}$ and a luminosity for energies not lower than $95 \%$ of the nominal of $5.3 \times 10^{32} \mathrm{~cm}^{-2} \mathrm{~s}^{-1}$. In order to go beyond the target value of $10^{33}$ at constant repetition rate, one would need either more particles per bunch (thereby increasing the background) or a minimum of two bunches per beam. Reaching sufficient luminosity with lower background might require four bunches per beam with a population reduced to $4.24 \times 10^{9}$ particles per bunch, say. This is the motivation behind the study of multibunch dynamics for CLIC.

The first step in this study is to consider the interaction of two bunches only, through the long-range wake fields induced by the first one. This would not only answer the stability question for two bunches, but also give useful information for a longer train if one assumes that the effect of the wake field generated by each bunch is mainly experienced by its immediate downstream neighbour. This assumption of nearest-neighbour coupling remains to be verified in the specific case of dipole-mode detuning with the CLIC acceleration structure. Nevertheless, one expects interesting indications on bcam stability from a two-bunch model, as explained below, knowing that the number of bunches per CLIC beam would always be small anyway.

\section{MULTIBUNCH MOTION FORMULATION}

Multibunch motion with nearest-neighbour coupling was recently formulated for smooth focusing [1], starting from the basic equation of motion

$$
\gamma(s) x^{\prime \prime}+\gamma^{\prime}(s) x^{\prime}+\gamma(s) k^{2} x=\int \rho W_{\mathrm{T}}^{\delta} x(s) \mathrm{d} z^{*}
$$

where $\gamma(s)$ is the energy (assumed to increase linearly with distance $s$ like $\left.\gamma=\gamma_{0}+G s\right), k$ the transverse wave number $(=1 / \beta), p$ the line charge density and $W_{T}^{\delta}$ the transverse wake field Green's function. To solve the problem, the author [1] looks for an equivalent equation of motion, reduced to one for zero acceleration. Deriving it from (1) requires a change of variables that cancels the s-dependence of $\gamma$ in the terms $\gamma x^{\prime \prime}$ and $\gamma k^{2} x$. This implies, because of the second derivative, a change of the independent coordinate identical to the change of $k$, while the amplitude $x$ must be normalized with the $\beta$ function that is scaled with $\gamma$ as $1 / k$, i.e.

$$
\mathrm{d} z=\frac{k}{k_{0}} \mathrm{~d} s=\left(\frac{\gamma_{0}}{\gamma}\right)^{\alpha_{\mathrm{b}}} \mathrm{d} s \quad x_{n}=x\left(\frac{\gamma}{\gamma_{0}}\right)^{\frac{\alpha_{\mathrm{b}}-1}{2}}
$$

The exponent $\alpha_{b}$ is an arbitrary coefficient that depicts the $\gamma$-scaling of the betatron function retained [3]. The transformation (2) does not exactly reduce Eq. (1) to one for constant $\gamma$ and the approximation that the relative energy gain $G / \gamma$ is small within a betatron period has to be invoked. Using Eq. (2), the length of the equivalent zero-acceleration linac is given for an arbitrary lattice scaling [3] by

$$
L=\int_{0}^{s_{\mathrm{tat}}}\left(\frac{\gamma_{0}}{\gamma}\right)^{\alpha_{\mathrm{b}}} \mathrm{d} s=\frac{1}{\left(1-\alpha_{\mathrm{b}}\right) G}\left[\gamma_{\mathrm{f}}^{1-\alpha_{\mathrm{b}}} \gamma_{0}^{\alpha_{\mathrm{b}}}-\gamma_{0}\right] \text {, }
$$

where $G$ is the energy gain, $\gamma_{0}$ and $\gamma_{\mathrm{f}}$ the initial and final energies. In the last proposal for CLIC, $\alpha_{b} \cong 0.4$ [3] and the length becomes equal to $1.2 \mathrm{~km}$ about.

The assumption that the wake field is independent of position along the linac allows for the derivation of an exact solution to the equations of motion [1]. The key point is that the amplitude growth is characterized by a power series in $f \cdot z$ and the condition to keep the bunch amplitudes small is

$$
f \cdot L<1 \quad \text { with } f=\frac{W_{\mathrm{Tb}} \mathrm{Ne} \beta_{0}}{2 E_{0}}
$$

where $W_{\mathrm{Tb}}$ is the wake-field strength at one bunch separation, $\mathrm{Ne}$ the charge per bunch, $\beta_{0}$ the initial betatron amplitude and $E_{0}$ the injection energy. For the $500-\mathrm{GeV}$ CLIC option 
parameters $\left(N=6 \times 10^{9}, \beta_{0} \cong 6.5 \mathrm{~m}, E_{0}=9 \mathrm{GeV}\right)$, condition (4) tells us that $W_{\mathrm{Tb}}$ must be smaller than $2.4 \mathrm{MV} / \mathrm{m}^{2} / \mathrm{nC}$ for the stability. Since the maximum transverse field $\hat{W}_{\mathrm{T}}$ is about $1100 \mathrm{MV} / \mathrm{m}^{2} / \mathrm{nC}$ in the tail of each bunch [4], this criterion implies an attenuation of approximately 450 of the field at one bunch separation. With a reduced population of $4.2 \times 10^{9}$ particles, an attenuation of 300 , say, is required according to (4), for multibunch stability. This already gives a valuable clue about the required reduction of the dipole modes by cell detuning, which remains to be cross-checked with numerical simulations.

\section{MULTIBUNCH MOTION SIMULATIONS}

The analytical formulation quoted in Section II, leading to a simple stability criterion, is valid for a constant-acceleration linac with smooth focusing and wake fields independent of position along the linac. Although the formalism has been extended to include linac FODO arrays and wake-field variations in the structures [1], numerical simulations have been retained for investigating further the two-bunch motion in an AG focusing lattice of the CLIC linac.

The tracking has been performed with two identical bunches, experiencing the same short-range wake fields whose maximum amplitude $\hat{W}_{\mathrm{I}}$ has been evaluated from 218 transverse modes [4]. In addition, a long-range wake field $W_{\mathrm{Tb}}$ has been superimposed in the second bunch, taking it constant within the bunch for simplification. The focusing FODO structure incorporates the most recent lattice scaling with energy [3] that allows, in the single-bunch mode, alignment tolerances of $50 \mu \mathrm{m}$ for quadrupoles, $10 \mu \mathrm{m}$ for cavities and 2 $\mu \mathrm{m}$ for beam-position monitors. Microwave quadrupoles are used for BNS damping and autophasing in each bunch.

The procedure for controlling the trajectory is based on the presence of monitors at each girder and possible iterations of the correction [3]. In the two-bunch mode however, the sequence of the successive operations is more complex. One admits the trajectory is first measured and minimized with one bunch only, with sets of RF quadrupoles adjusted for stabilizing transverse oscillations. After an optimum correction, two bunches are injected in the linac, and the trajectory mcasurcd using the total integrated signal of both bunches is corrected. During this second operation, RF quadrupoles are tuned again by sectors (700-m long, typically), and iterations are carried out whenever it is worthwhile for emittance control.

Simulations were started with the lower bunch population considered $\left(N=4.2 \times 10^{9}\right)$ for different $\hat{W}_{\mathrm{T}} / W_{\mathrm{Tb}}$ ratio. It has been observed that beam breakup always rises strongly over a short distance $(200 \mathrm{~m}$, say), after a long and slow buildup of coherent instability (Fig. 1). The physical explanation for such a latent instability has already been proposed in the past [5]. After the initial perturbation, both bunches oscillate with the same phase and the wake field excitation is in quadrature with the amplitude of the oscillation. Wake fields provoke then a mere phase slip that increases with the distance travelled by the bunches. The oscillations of the second bunch lag behind those of the first and their amplitude spirals slowly towards larger values over a high number of betatron wavelengths depending on field strength. Eventually, when a finite phase lag develops, an in-phase excitation component appears, leading to a rapid amplification of the initial amplitude. For $W$-ratio of 50, this amplification takes place within the $3-\mathrm{km}$ length of the $250-\mathrm{GeV}$ CLIC linac and for 100 , within the 12-km length of the 1-TeV CLIC linac (Fig. 1). In order to have it beyond the exit of the $1-\mathrm{TeV}$ linac, a wake-field ratio of at least 150 is required with this bunch intensity. Having pushed the beam breakup outside the linac, RF quadrupoles can be adjusted to keep the emittance dilution low over the linac length and fight the coherent instability of bunch 2 . For that purpose, RFQ strength is reduced, mainly towards the end of the linac, in order to compromise between BNS damping of short-range wake field in both bunches and counteracting long-range constant wake field in bunch 2 . This succeeded in limiting emittance growth to tolerable values (average of $2 \times$ $10^{-7} \mathrm{rad} \mathrm{m}$ ) for $W$-ratio equal to 100 (Fig. 2) and 300 (Fig. 3).

Considering then a bunch population of $6 \times 10^{9}$, a $W$-ratio of at least 500 is necessary, in order to obtain final normalized emittances (Fig. 4) that lead to about twice the luminosity expected with $\gamma \varepsilon_{y}=2 \times 10^{-7} \mathrm{rad} \mathrm{m}$ and one bunch per beam.

\section{COMMENTS AND PERSPECTIVE}

While the analytical formulation suggests a wake-field attenuation on the level of the follower-bunch by 300 and 450 depending on the population, two-bunch tracking shows that careful adjustment of the RF quadrupoles makes it possible to keep both bunches stable within requirements with factors 100 and 500 , i.e. in fairly good agreement. These $W$-ratios look high, however, in the sense that they are difficult to achieve by detuning the dipole modes in the $30-\mathrm{GHz}$ cells, as recent studies show [6]. A possible way out could be the introduction of two dipole modes at different frequencies in every staggered, tuned accelerating section or the introduction of two different types of sections, each with one dipole mode, the two modes being separated by $\pi$ at the second bunch position. As suggested by W. Schnell, alternating these sections to have a succession of opposite wake-field amplitudes corresponds to a pseudolocal compensation. Tracking with $W$-amplitudes roughly opposed in two contiguous sections, but with $20 \%$ systematic difference and $10 \%$ r.m.s. spread, indicates it is possible in a particular case to obtain the target-emittances at the $250-\mathrm{GeV}$ linac end with a $\hat{W}_{\mathrm{T}} / W_{\mathrm{Tb}}$ ratio of 40 (Fig. 5). This result and the phenomenology of the latent instability (Section III) suggest the further investigation of what I would call alternate wake-kick damping. 


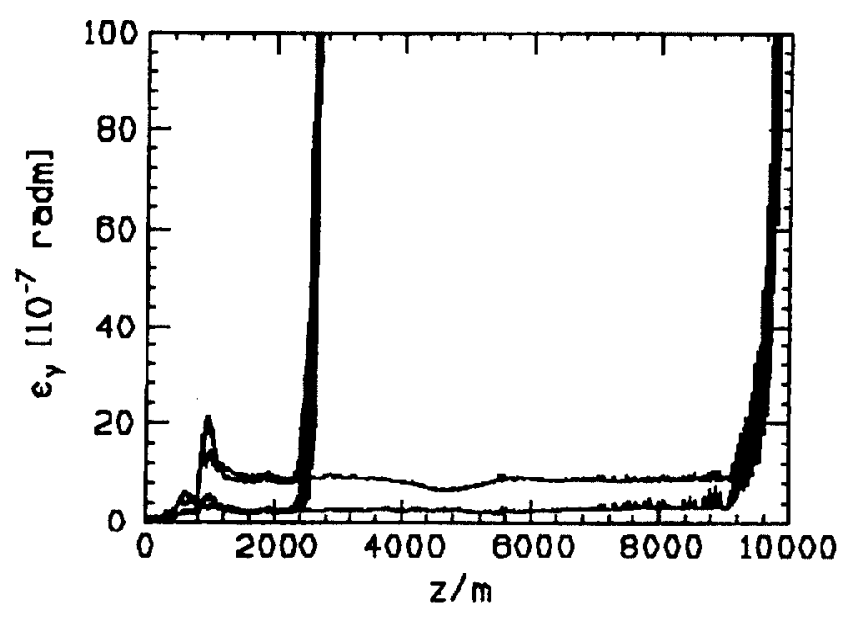

Fig. 1: Beam breakup due to long-range wake fields, with $W$-ratio of 50 and 100

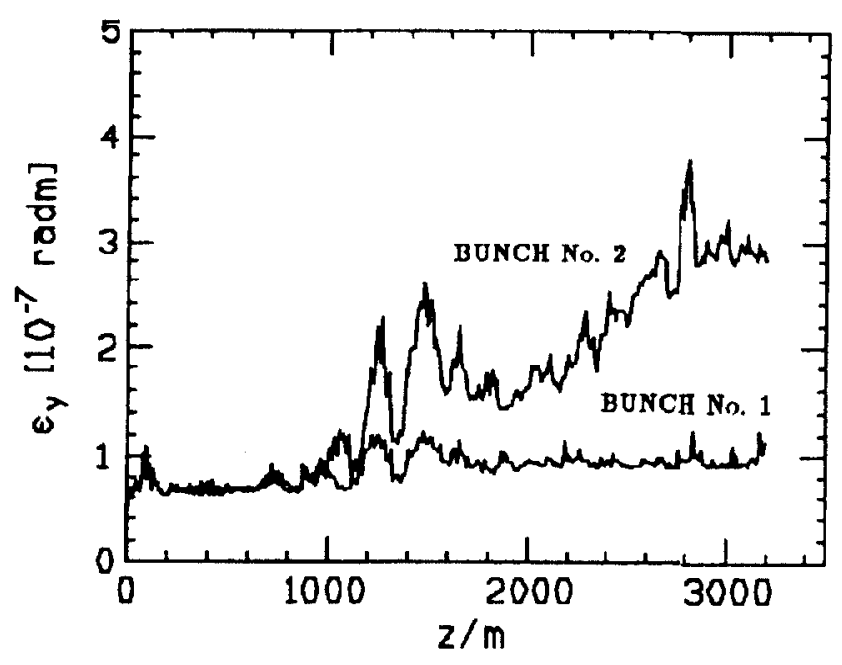

Fig. 2: Emittance growth with $N=4.2 \cdot 10^{9}$ and $W$-ratio of 100

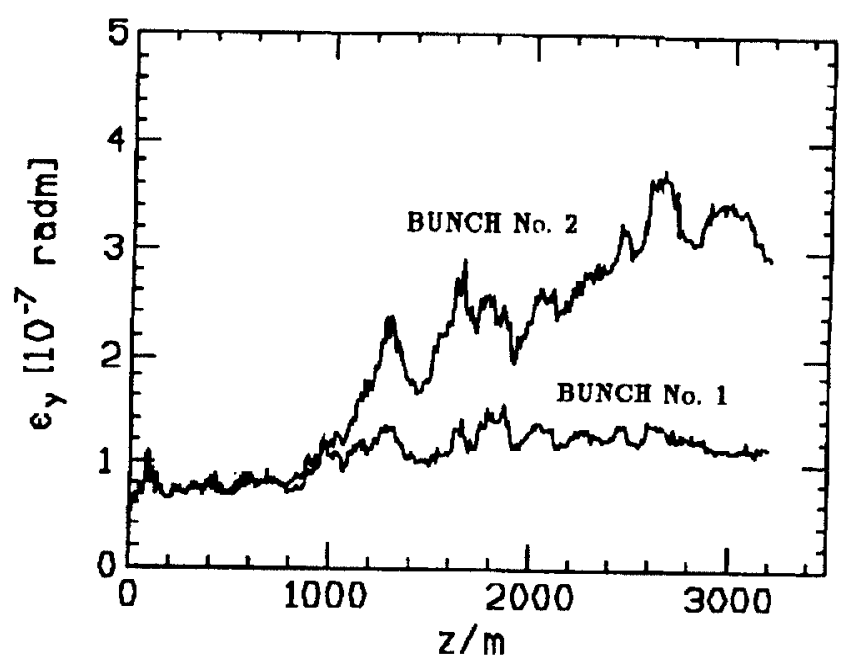

Fig. 3: Emittance growth with $N=4 \cdot 2 \cdot 10^{9}$ and $W$-ratio of 300

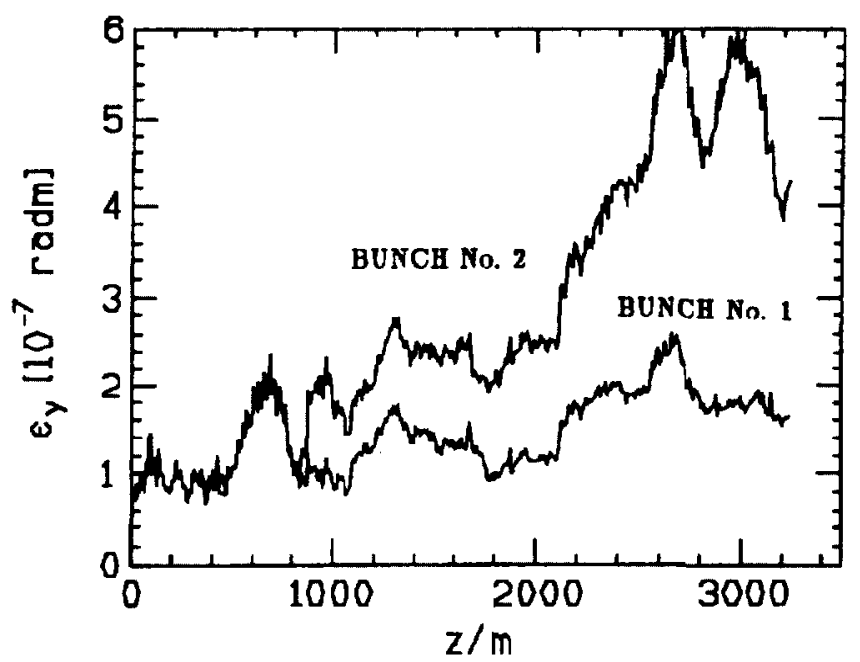

Fig. 4: Emittance growth with $N=6 \cdot 10^{9}$ and $W$-ratio of 500

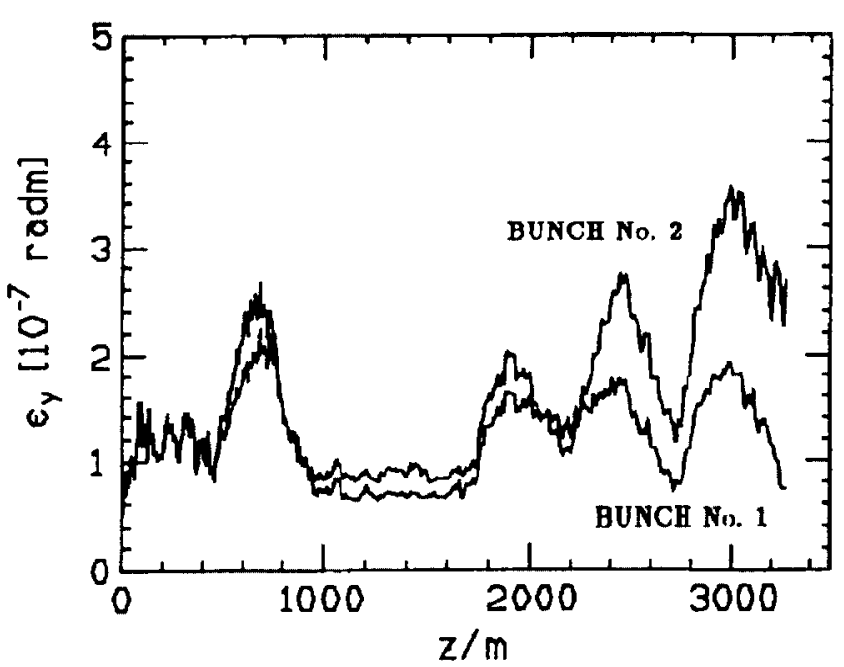

Fig. 5: Two-bunch emittance growth with alternate wake-kick damping, $N=6 \cdot 10^{9}$ and $W$-ratio of 40

\section{REFERENCES}

[1] C. Adolphsen, "Multibunch motion with nearest-neighbor wake-field coupling," XVth Int. Conf. H. E. Acc., Hamburg, 1992.

[2] G. Guignard, "Beam stability simulations in CLIC linacs," XVth Int. Conf. H. E. Acc., Hamburg, 1992.

[3] G. Guignard, "Lattice scaling and emittance control in the CLIC main linac," this Conference.

[4] A. Millich, "Computation of wake fields for the CLIC waveguide," European Part. Acc. Conf., Berlin, 1992.

[5] H. Henke and W. Schnell, "Analytical criterion for the onset of transverse damping," CERN-LEP-RF/86-18, 1986.

[6] I. Wilson, W. Wuensch, "Multi-bunch studies for the CERN Linear Collider," this Conference. 